\title{
ON THE RELATIVE LENGTHS OF SIDES OF CONVEX POLYGONS
}

\author{
ZSOLT LÁNGI
}

\begin{abstract}
Let $C$ be a convex body. By the relative distance of points $p$ and $q$ we mean the ratio of the Euclidean distance of $p$ and $q$ to the half of the Euclidean length of a longest chord of $C$ parallel to $p q$. The aim of the paper is to find upper bounds for the minimum of the relative lengths of the sides of convex hexagons and heptagons.
\end{abstract}

\section{INTRODUCTION}

Let $p q$ be the closed segment with endpoints $p$ and $q$ in the Euclidean plane $E^{2}$. Denote by $|p q|$ the Euclidean length of $p q$. Take a convex body $C \subset E^{2}$. Consider a chord $p^{\prime} q^{\prime}$ of $C$ such that there is no longer chord in $C$ parallel to $p q$. The ratio of $|p q|$ to $\frac{1}{2}\left|p^{\prime} q^{\prime}\right|$ is called the $C$-distance of $p$ and $q$ and it is denoted by $d_{C}(p, q)$. By the $C$-length of a closed segment we mean the $C$-distance of its endpoints. If it is obvious which convex body we refer to, we may use the names relative distance or relative length. We call a side of a convex $n$-gon relatively short (respectively, relatively long) if its relative length is not greater (not smaller) than the relative length of a side of the regular $n$-gon.

Doliwka and Lassak [2] proved that every convex pentagon has a relatively short and a relatively long side. As the relative length of the sides of a regular hexagon is 1 , the analogous qustion about hexagons is whether every convex hexagon has a side of relative length at least 1 , and a side of relative length at most 1 . Doliwka and Lassak [2] presented the following examples that the answer for the above question is negative.

Consider the hexagon $H_{0}$ which is the convex hull of a regular triangle and its homothetic copy with the homothety centre in the centre of gravity of the triangle with the homothety ratio $1-\sqrt{3}$. The relative length of the sides of this hexagon is $8-4 \sqrt{3} \approx 1.071>1$. The mentioned authors conjecture that every convex hexagon has a relatively long side and a side of relative length at most $8-4 \sqrt{3}$. The first part of this conjecture follows from the paper [1] of Chakerian and Talley. The aim of the present paper is to prove the second part of this conjecture. We also show that every convex heptagon has a side of unit relative length.

\section{HeXAGOns}

Theorem 1. Every convex hexagon has a side of relative length at most $8-4 \sqrt{3}$.

Our proof of this theorem is based on two lemmas.

1991 Mathematics Subject Classification. 52A10, 52A40, 52C15.

Key words and phrases. convex body, relative distance. 
Lemma 1. Let $G$ be a convex $n$-gon, where $n \geq 6$. Assume that a triangle of the largest possible area inscribed in $G$ has a side which coincides with a side of $G$. Then $G$ has a side of $G$-length at most 1 .

Proof. Let $T=a b c$ be a triangle mentioned in the formulation of our lemma. Observe that we can assume that $a b$ is a side of $G$, and $c$ is a vertex of $G$. At least one of the two pieces of the boundary of $G$ between $a$ and $c$ contains at least two additional vertices $e$ and $f$ of $G$. For instance, let $e$ be between $c$ and $f$ on this piece.

Since the ratio of the areas of two figures does not change under affine transformations, we may assume in our proof that $a b c$ is an isosceles triangle with right angle at $b$. Take the point $d$ such that $S=a b c d$ is a square. Since $a b c$ is a triangle of maximal area, we conclude that $e$ and $f$ belong to $S$.

Consider the convex pentagon $P=a b c e f$. First, we intend to show that at least one of the relative distances $d_{P}(c, e), d_{P}(e, f), d_{P}(f, a)$ is at most 1 . We dissect $S$ into four equal squares $S_{a}, S_{b}, S_{c}, S_{d}$ containing $a, b, c, d$, respectively. Since $G$ is convex, $e$ and $f$ are not in the interior of $T$. If $d_{P}(c, e)>1$ and $d_{P}(f, a)>1$, then $e \notin S_{c}$, and $f \notin S_{a}$ and thus $e \in S_{d}$ and $f \in S_{d}$. Hence $d_{P}(e, f) \leq 1$. We see that at least one of the numbers $d_{P}(c, e), d_{P}(e, f), d_{P}(f, a)$ is at most 1 .

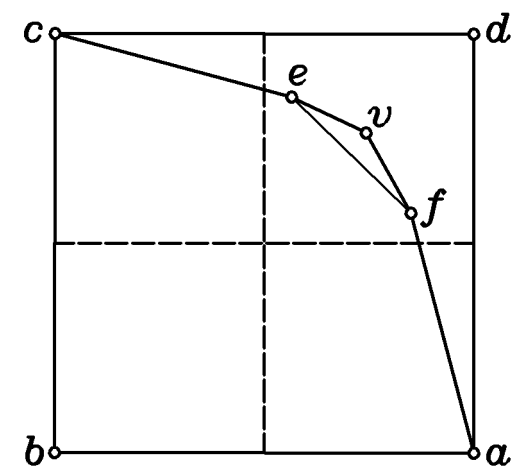

Figure 1

Finally, we intend to show that if one of the mentioned $P$-distances is at most 1 , then $G$ has a side of $G$-length at most 1 . We assume that $d_{P}(e, f) \leq 1$ (analogical consideration can be applied for the remaining two cases). Examine the case when $e$ and $f$ are consecutive vertices of $G$. Since $\mathrm{P}$ is a subset of $G$, we have $d_{G}(p, q) \leq$ $d_{P}(p, q)$ for arbitrary points $p, q$. Thus, in this case the thesis of our lemma holds true. Take into account the opposite case, when $e$ and $f$ are not consecutive vertices, and take a vertex $v$ of $G$ between them. Let $V$ be a side of $G$ with endpoint $v$. Consider the chords $C_{a}$ and $C_{c}$ of $G$ with endpoints $a$ and $c$, respectively, which are parallel to $V$. Observe that $C_{a}$ or $C_{c}$ is at least twice as long as $V$. Hence, the $G$-length of $V$ is at most 1 .

Lemma 2. Consider a convex hexagon $H=$ abcdef such that the triangle ace is regular. Let us take the lines through a, c, e parallel to the segments ce, ea, ac, respectively. The intersections of these lines are denoted by $a_{0}, c_{0}, e_{0}$ (they are opposite to $a, c, e$, respectively). Assume that $b, d, f$ are in the triangle $a_{0} c_{0} e_{0}$ and that the angles cab $\angle$, acb $\angle$, aef $\angle$, eaf $\angle$ are equal $\alpha$. Denote the angle ecd $\angle$ by $\beta$, and denote the angle ced $\angle$ by $\gamma$. If $0<\alpha<\frac{\pi}{6}, 0<\min (\beta, \gamma)<\frac{\pi}{6}, d_{H}(c, d) \geq$ $8-4 \sqrt{3}$, and $d_{H}(d, e) \geq 8-4 \sqrt{3}$, then $\min (\beta, \gamma) \geq \alpha$ with equality if and only if 
Proof. We choose a Cartesian coordinate system such that $a, c, e$ are $(0,0),(1, \sqrt{3})$ and $(-1, \sqrt{3})$, respectively. Since $d_{H}(d, e) \geq 8-4 \sqrt{3}$, then $d$ is not in the interior of the homothetic copy $C_{1}$ of the quadrangle ce $f a$ with the homothety ratio $-(4-2 \sqrt{3})$ such that the image of $c$ is $e$. Moreover, also $d$ is not in the interior of the homothetic copy $C_{2}$ of the quadrangle $e a b c$ with the homothety ratio $-(4-2 \sqrt{3})$ such that the image of $e$ is $c$. The boundaries of $C_{1}$ and $C_{2}$ inside of the triangle $c a_{0} e$ intersect each other at one point. Denote it hv $d$.

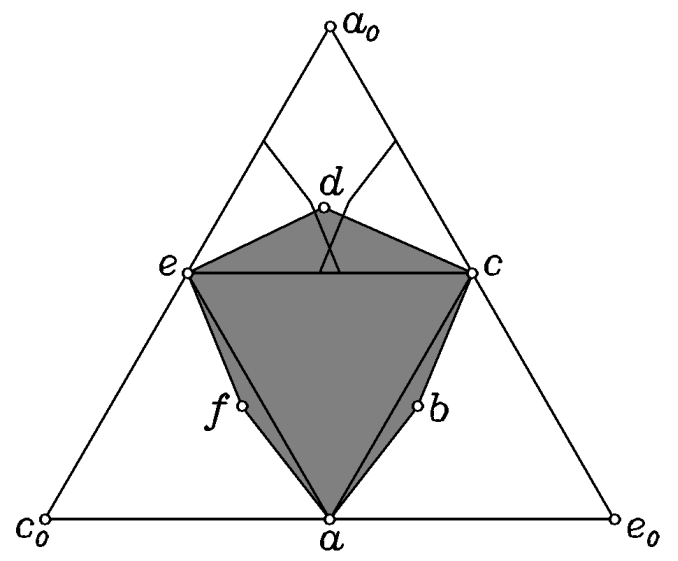

Figure 2

Case 1 , when $d_{0}$ is on the images of the sides ef and $b c$.

The minimum of $\beta$ and $\gamma$ is attained for $d=d_{0}$. The ordinate of $d_{0}$ is

$$
\tan \left(\alpha+\frac{\pi}{3}\right)(7-4 \sqrt{3})+\sqrt{3}
$$

This implies the inequality

$$
\tan (\min (\beta, \gamma)) \geq \tan \left(\alpha+\frac{\pi}{3}\right)(7-4 \sqrt{3})
$$

But it can be easily verified that if $0<\alpha<\frac{\pi}{6}$, then $\tan (\alpha) \leq \tan \left(\alpha+\frac{\pi}{3}\right)(7-4 \sqrt{3})$ with equality if and only if $\alpha=\frac{\pi}{12}$. Hence, the equality can hold if and only if $\alpha=\min (\beta, \gamma)=\frac{\pi}{12}$. But when $\beta$ or $\gamma$ is equal to $\frac{\pi}{12}, d_{0}$ is the only point on the segment in the triangle $e c a_{0}$ determined by the angle $\frac{\pi}{12}$ which is not in the interiors of $C_{1}$ and $C_{2}$. That is, we have $\beta=\gamma=\frac{\pi}{12}$.

Case 2: when $d_{0}$ is on the images of the sides $f a$ and $a b$.

We get the minimum of $\beta$ when $d$ is $d_{0}$ or when $d$ is the homothetic image of $a$ in the homothetic copy of the quadrangle cefa. Hence $\beta \geq \frac{\pi}{6}$. A similar inequality holds for $\gamma$. Therefore $\min (\beta, \gamma) \geq \frac{\pi}{6}$, contrary to the hypothesis.

Proof of Theorem. Consider a convex hexagon $H=$ abcdef. If a triangle of the largest possible area inscribed in $H$ has a side which coincides with a side of $H$, then we apply Lemma 1.

Let us look to the opposite case when every triangle of the maximum area inscribed in $H$ does not contain a side of $H$. Observe that then ace or bdf is a triangle of maximal area. Consider the first possibility (in the other one, further consideration is analogical). Since the relative distance is affine invariant, we can assume that ace is a regular triangle with vertices $a(0,0), c(1, \sqrt{3}), e(-1, \sqrt{3})$ in a 
$e$ parallel to the segments $c e, e a, a c$, respectively. Denote the point of intersection of $L_{c}$ and $L_{e}$ by $a_{0}$. Similarly, let $c_{0}$ be the intersection of $L_{a}$ and $L_{e}$. Moreover, let $e_{0}$ be the intersection of $L_{a}$ and $L_{c}$. Since ace is a triangle of maximum area inscribed in $H$, the points $b, d$ and $f$ belong to the triangle $a_{0} c_{0} e_{0}$. Denote the angles $c a b \angle, a c b \angle$, ecd $\angle, \ldots$, eaf $\angle$ by $\alpha_{1}, \alpha_{2}, \ldots \alpha_{6}$, respectively.

In order to prove our theorem we intend to show that if the relative lengths of the sides of $H$ are at least $8-4 \sqrt{3}$, then $\alpha_{i}=\frac{\pi}{12}$ for $i=1, \ldots, 6$.

In further consideration we exclude the case when $\alpha_{i}=0$ for a certain $i$ because in this special situation the hexagon contains a closed segment containing three consecutive vertices which means that it has a side of relative length at most 1.

Assume that $\alpha_{4}=\min \left\{\alpha_{1}, \ldots, \alpha_{6}\right\}$.

Case 1 , when $\alpha_{4}<\frac{\pi}{6}$.

Consider first an auxiliary hexagon $H^{\prime}$ in which we have $\alpha_{4}$ in the place of $\alpha_{1}, \alpha_{2}, \alpha_{5}, \alpha_{6}$. Then, from $H^{\prime} \subset H$ we get that $d_{H}(c, d) \leq d_{H^{\prime}}(c, d)$ and $d_{H}(d, e) \leq$ $d_{H^{\prime}}(d, e)$.

Now we apply Lemma 2 for $H^{\prime}$ putting $\alpha_{3}$ in the part of $\beta$, and $\alpha_{4}$ in the part of $\alpha$ and $\gamma$. We get that $\alpha_{4} \leq \alpha_{4}$ with equality if and only if $\alpha_{3}=\alpha_{4}=\frac{\pi}{12}$. Since $\alpha_{4}$ is the minimal angle from among $\alpha_{1}, \ldots, \alpha_{6}$, all those angles are at least $\frac{\pi}{12}$. Let us take the homothetic copies of the quadrangles cefa and eabc with homothetic ratio $-(4-2 \sqrt{3})$ such that the images of $c$ and $e$ are $e$ and $c$, respectively. Since $d$ is in the interior of neither of the two copies, we get that $\min \left(\alpha_{1}, \alpha_{2}\right)$ and $\min \left(\alpha_{5}, \alpha_{6}\right)$ are at most $\frac{\pi}{12}$. Consequently they are equal to $\frac{\pi}{12}$. Now we take an auxiliary hexagon $H^{\prime \prime}$ in which $\alpha_{5}$ and $\alpha_{6}$ are replaced by $\alpha_{4}=\frac{\pi}{12}$. We apply Lemma 2 for $H^{\prime \prime}$ and we get that $\frac{\pi}{12} \leq \frac{\pi}{12}$ with equality if and only if $\alpha_{1}=\alpha_{2}=\frac{\pi}{12}$. Thus, we can apply Lemma 2 for $H$, and as a result we get that $\alpha_{i}=\frac{\pi}{12}$ for every $i \in\{i=1,2, \ldots, 6\}$.

It can be easily verified that this hexagon is nothing else but the hexagon $H_{0}$ mentioned in Introduction.

Case 2, when $\alpha_{4} \geq \frac{\pi}{6}$.

According to our previous assumption about $\alpha_{4}$, all the angles are at least $\frac{\pi}{6}$. Notice that in this case the area of the triangle $b d f$ is not less than the area of the triangle ace, with equality if and only if all the six angles are $\frac{\pi}{6}$. Hence this case concerns only the regular hexagon, the relative length of the sides of which is equal to 1 .

The proof of Theorem also shows that the only hexagons such that the relative lengths of its sides are at least $8-4 \sqrt{3}$ are the affine images of the hexagon $H_{0}$ constructed by Doliwka and Lassak.

\section{Heptagons}

Corollary. Every convex heptagon has a side of relative length at most 1.

Proof. Let $H=a b c d e f g$ be a convex heptagon, such that all the relative lengths of its sides are greater than 1. According to Lemma 1 we can assume that acf is a triangle of maximal area inscribed in $H$. As relative distance is affine invariant, we can assume that the triangle $a c f$ is regular. Let us take a Cartesian coordinate system such that $a, c$, and $f$ are $(0,0),(1, \sqrt{3}),(-1, \sqrt{3})$, respectively. We define the points $a_{0}, c_{0}, f_{0}$ similarly like in the proof of Theorem. Since $a c f$ is a triangle 
of the segments $c f, a_{0} f$ and $a_{0} c$, respectively. As $d_{H}(c, d)$ and $d_{H}(e, f)$ are greater than 1, the points $d$ and $e$ belong to the rhombus $a^{\prime} f^{\prime} a_{0} c^{\prime}$. The convexity of $H$ implies that the slope of the segment $d e$ is between $-\sqrt{3}$ and $\sqrt{3}$. Hence $d_{H}(d, e) \leq$ 1. But this contradicts the assumption that the relative lengths of all the sides of $H$ are greater than 1 .

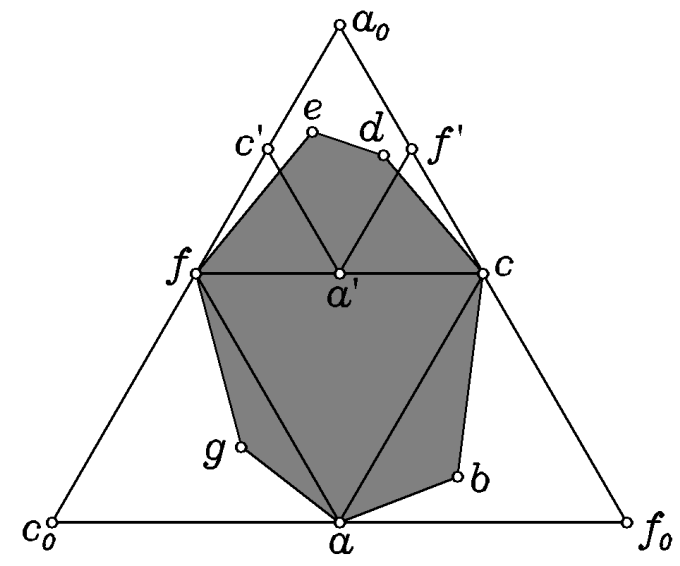

Figure 3

The example of the degenerated heptagon with four vertices in the vertices of a square and with three remaining vertices in the midpoints of the sides of the square shows that this result is the best possible.

Acknowledgements. I am indebted to M. Lassak and E. Makai for helpful comments.

\section{REFERENCES}

1. G. D. Chakerian, W. K. Talley, Some properties of the self-circumference of convex sets, Arch. Math. 20 (1969), 431-443.

2. K. Doliwka, M. Lassak, On relatively short and long sides of convex pentagons, Geom. Dedicata 56 (1995), 221-224.

ZSOLT LÁNGI

Polytechnic of Dunaújváros

Institute of Natural Sciences

H-2400 DunAúJVÁros

TÁnCSics M. Út 1/A, Hungary

E-MAIL:ZSLANGI@KAC.POLIOD.HU 